\title{
Novel biomarkers and age-related metabolite correlations in plasma and dried blood spots from patients with succinic semialdehyde dehydrogenase deficiency
}

Trevor Kirby ${ }^{1 \dagger}$, Dana C. Walters ${ }^{1+}$, Xutong Shi ${ }^{1}$, Coleman Turgeon ${ }^{2}$, Piero Rinaldo ${ }^{2}$, Erland Arning ${ }^{3}$, Paula Ashcraft ${ }^{3}$, Teodoro Bottiglieri ${ }^{3}$, Melissa DiBacco ${ }^{4}$, Phillip L. Pearl ${ }^{4}$, Jean-Baptiste Roullet ${ }^{1}$ and K. Michael Gibson ${ }^{1 *}$

\begin{abstract}
Background: Previous work has identified age-related negative correlations for $\gamma$-hydroxybutyric acid (GHB) and $\gamma$ aminobutyric acid (GABA) in plasma of patients with succinic semialdehyde dehydrogenase deficiency (SSADHD). Using plasma and dried blood spots (DBS) collected in an ongoing natural history study, we tested the hypothesis that other biomarkers would follow a similar age-related negative correlation as seen for GHB/GABA. Samples (mixed sex) included: patients ( $n=21$ unique samples, $1-39.5$ yrs) and parallel controls ( $n=9$ unique samples, 8.4$34.8 \mathrm{yrs}$ ). Archival control data (DBS only; $n=171,0.5-39.9 \mathrm{yrs}$ ) was also included.

Results: Metabolites assessed included amino acids (plasma, DBS) and acylcarnitines, creatine, creatinine, and guanidinoacetate (DBS only). Age-related negative correlations for glycine (plasma, DBS) and sarcosine (Nmethylglycine, plasma) were detected, accompanied by elevated proline and decreased levels of succinylacetone, argininosuccinate, formaminoglutamate, and creatinine. Significantly low acylcarnitines were detected in patients across all chain lengths (short-, medium- and long-chain). Significant age-dependent positive correlations for selected acylcarnitines (C6-, C12DC(dicarboxylic)-, C16-, C16:1-, C18:1-, C18:2OH-carnitines) were detected in patients and absent in controls. Receiver operating characteristic (ROC) curves for all binary comparisons revealed argininosuccinate and succinylacetone to be the most discriminating biomarkers (area > 0.92).

Conclusions: Age-dependent acylcarnitine correlations may represent metabolic compensation responsive to agerelated changes in GHB and GABA. Our study highlights novel biomarkers in SSADHD and expands the metabolic pathophysiology of this rare disorder of GABA metabolism.
\end{abstract}

Keywords: Dried blood spots, Amino acids, Acylcarnitines, Creatinine, guanidinoacetate, Age-dependent correlations, $Y$-Hydroxybutyrate (GHB), $\gamma$-Aminobutyrate (GABA)

\footnotetext{
* Correspondence: mike.gibson@wsu.edu

${ }^{\dagger}$ Trevor Kirby and Dana C. Walters contributed equally to this work.

'Department of Pharmacotherapy, College of Pharmacy and Pharmaceutical Sciences Building Room 210C, Washington State University, 412 E. Spokane Falls Boulevard, Spokane, WA 99202-2131, USA

Full list of author information is available at the end of the article
}

(c) The Author(s). 2020 Open Access This article is licensed under a Creative Commons Attribution 4.0 International License, which permits use, sharing, adaptation, distribution and reproduction in any medium or format, as long as you give appropriate credit to the original author(s) and the source, provide a link to the Creative Commons licence, and indicate if changes were made. The images or other third party material in this article are included in the article's Creative Commons licence, unless indicated otherwise in a credit line to the material. If material is not included in the article's Creative Commons licence and your intended use is not permitted by statutory regulation or exceeds the permitted use, you will need to obtain permission directly from the copyright holder. To view a copy of this licence, visit http://creativecommons.org/licenses/by/4.0/ The Creative Commons Public Domain Dedication waiver (http://creativecommons.org/publicdomain/zero/1.0/) applies to the data made available in this article, unless otherwise stated in a credit line to the data. 


\section{Background}

Succinic semialdehyde dehydrogenase (SSADH) deficiency (SSADHD) is a rare disorder on the GABA metabolic pathway. The product of glutamate decarboxylation, GABA is catabolized to succinic acid in a two-enzyme sequence, including generation of succinic semialdehyde (SSA) catalyzed by GABA-aminotransferase and the further oxidation of SSA to succinic acid catalyzed by SSADH [10]. The backbone of GABA thus enters the tricarboxylic acid cycle for further metabolism. SSADHD is frequently referred to as $\gamma$-hydroxybutyric aciduria. This is due to the fact that accumulated SSA, in the absence of functional SSADH activity, is converted to $\gamma$-hydroxybutyrate (GHB), the latter being a compound with diverse and still poorly understood neuromodulatory roles [13]. In addition to increased GABA and GHB, patients manifest a broad variety of additional metabolic abnormalities, which have recently been summarized $[2,11]$.

The phenotype of SSADHD encompasses developmental and speech delays, hypotonia, neuropsychiatric morbidity (attention deficit disorder, obsessive compulsive behavior), and seizures in $\sim 50 \%$ of patients. The metabolic signature includes elevated GHB and GABA in physiological fluids, among other metabolites [11]. The incidence of SSADHD is estimated to be $1: 10^{6}$, but expanding molecular genetic studies are likely to reveal a higher prevalence. To explore the clinical and metabolic evolution of SSADHD, we have recently embarked on an NIHfunded natural history study. Enrolling up to 55 patients (any age), this study encompasses multiple neuroimaging and electrophysiological evaluations over a 5-year period. An important ancillary component of clinical evaluation is the collection of biospecimens yearly, including plasma, white and red cells, DNA and RNA, hair, saliva, stool, urine, fibroblasts, and others. These samples, aliquoted and stored long-term, provide unique opportunities for future analyses as our knowledge of SSADHD evolves, and the potential to correlate biomarkers levels in different matrices with clinical features and disease severity.

Earlier studies in plasma and RBC samples derived from SSADHD patients identified age-dependent negative correlations for both GHB and GABA [10]. GHB in plasma reached a nadir at approximately the age of puberty $(\sim$ 13-16 years of age), while the same nadir for GABA occurred well into the 3rd decade of life. DiBacco et al. [6] observed an age-dependent association with worsening of epilepsy, obsessive-compulsive behavior, and sleep disturbances in an older SSADHD cohort. This suggests that declining GABA and GHB levels in peripheral biofluids might represent a metabolic correlate of adulthood symptom severity. Based on this rationale, we examined the hypothesis that other biomarkers of SSADHD (amino acids, acylcarnitines, etc.) would manifest an age-dependent negative correlation as seen for GHB and GABA.

\section{Results}

Significant elevations of sarcosine (sarc) and glutamate (glu), and a significant decrease in ethanolamine (EA), were demonstrated in SSADHD plasma (Fig. 1). Agedependent negative correlations were observed for glycine (gly) and sarc in patients, and absent in parallel controls (Fig. 2; control data not shown). The negative correlation for gly in patients was replicated in DBS (Fig. 2; sarc not measured in DBS). An age-dependent negative correlation was observed for serine (ser) in patients that mirrored a comparable age-dependent negative correlation in controls (data not shown). Based on the correlations for gly and ser, we correlated these amino acids in different matrices. In plasma, there were significant positive correlations for both control and SSADHD cohorts (Fig. 2). There was a significant correlation for gly and ser in control DBS, but not in the SSADHD cohort (Fig. 2), likely reflecting the negative age-dependent gly correlation in patients. In patient DBS, elevations of gly, alanine (ala), proline (pro) and phenylalanine were accompanied by significantly decreased ornithine (orn), argininosuccinate (ASA), glutamine (gln) and succinylacetone (Suac) (Fig. 3; Fig. 4 depicts ROC curves corresponding to Fig. 3).

Of thirty eight acylcarnitine species quantified, significant alterations were detected in twenty four. Twenty three included significant decreases in patients compared to control, with only C5:1 carnitine showing an elevation (Fig. 5). Decreased short chain acylcarnitines included C2-, C3-, formiminoglutamate (figlu), C4-, $\mathrm{C} 4 \mathrm{OH}, \mathrm{C} 5 \mathrm{OH}$ and $\mathrm{C} 6$ carnitines (Fig. 5; Fig. 6 depicts ROC curves corresponding to Fig. 5). Medium chain acylcarnitines that were significantly decreased in patient DBS included C8-, C8:1, C10- and C10:1 carnitines (Suppl. Fig. 1). In terms of long-chain acylcarnitines, significantly decreased levels in patients included C14-, C14:1, C14:2 and C14OH- carnitines (Suppl. Fig. 2), C16-, C16:1- and C16OH carnitines (Suppl. Fig. 3), and C18-, C18:1-, C18:2-, C18OH and C18:1OH-carnitines (Suppl. Fig. 4; Suppl. Fig. 5 depicts the ROC curves corresponding to Suppl. Fig. 4).

Based on results for gly and sarc, and earlier results for GABA and GHB, correlative analyses were examined as a function of age for all acylcarnitines in both patient and control cohorts. Significant age-related correlations were observed for C3-, C16:1OH- and C18:2-carnitines in patients DBS, but comparable correlations were observed in controls (data not shown). Conversely, significant agedependent positive correlations were observed for C6-, C12DC-, C16-, C16:1, C18:1 and C18:2OH carnitines in patients which were absent in controls (Fig. 7, Suppl. Fig. 6).

Patients with SSADHD displayed significant alterations in creatinine (crn) and guanidinoacetate (guac) in DBS without concomitant changes in creatine (cre) (Suppl. Fig. 7). Controls demonstrated significant age-related positive correlations for guac $(P<0.0001)$, cre $(P<0.05)$ and 


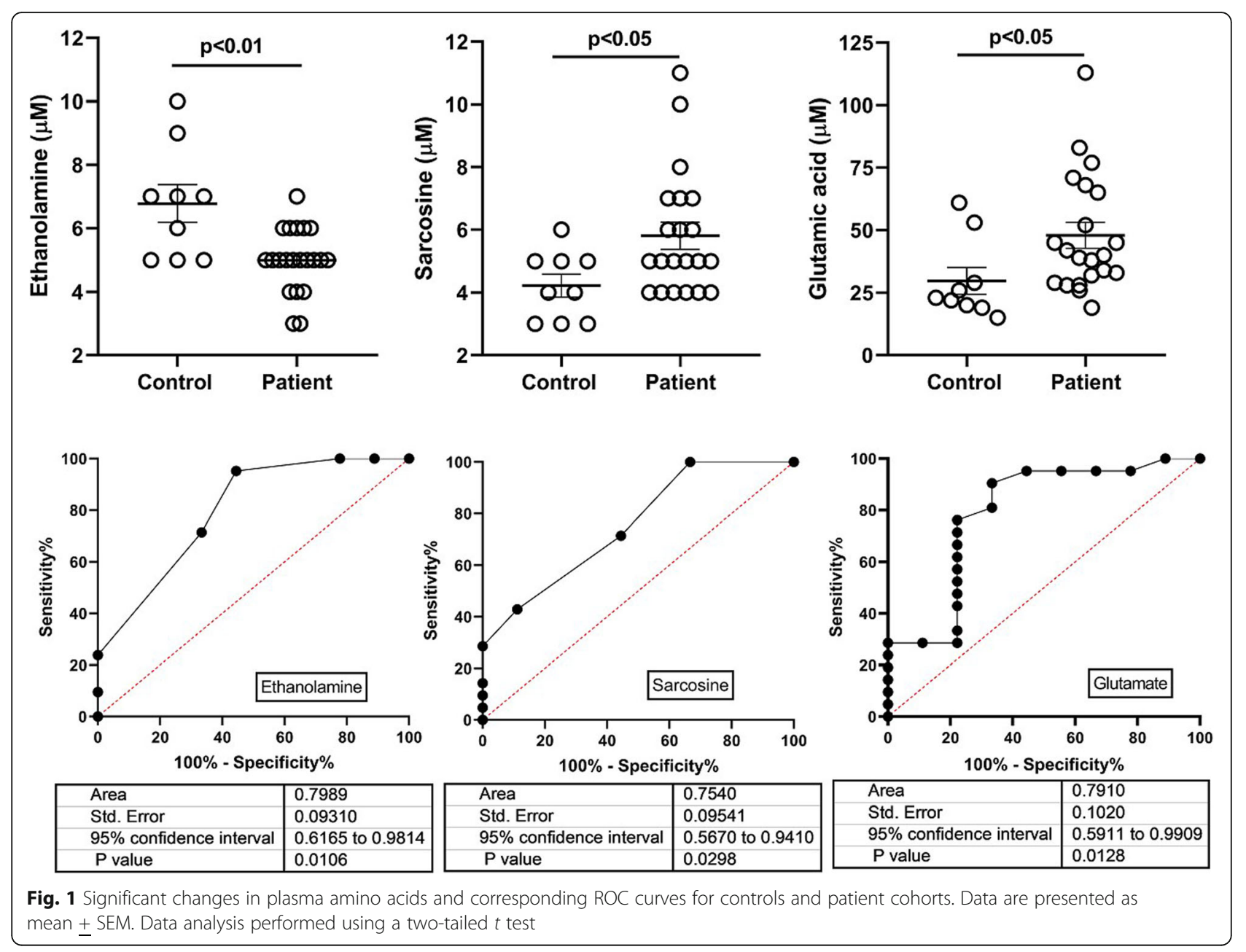

crn $(\mathrm{P}<0.0001)$, whereas patients demonstrated this correlation only for $\mathrm{crn}(\mathrm{P}<0.0001$, data not shown). Correlative analyses for all three metabolites were assessed in both cohorts. Controls demonstrated significant age-related positive correlations cre vs crn $(\mathrm{P}<0.0001)$, guac vs. crn $(\mathrm{P}<$ 0.0001 ), but no significant correlation for cre vs. guac (data not shown); conversely, patients demonstrated no correlations for the same comparisons (data not shown), although there was a modest trend for guac vs. $\operatorname{crn}(P=0.077$; data not shown).

\section{Discussion}

Gly and its $N$-methylated precursor, sarc, showed an agedependent negative correlation in patients, and the gly trend was confirmed in patient plasma and DBS. Like GABA, gly is an inhibitory neurotransmitter, and sarc is an inhibitor of the glycine type I transporter $[8,15]$. The nadirs for gly and sarc approximated the same time point (midteens, roughly the onset of puberty) as earlier observed for GHB [10]. Although both DBS and plasma demonstrated age-related negative correlations, the glycine level when directly comparing patients and controls was increased only in DBS and not plasma (Fig. 3). Consistent with the agerelated nadir for peripheral gly, we detected normal gly in autopsied brain from an adult SSADHD patient (37 years) [11]. Elevated plasma glutamate (glu), the GABA precursor, was demonstrated in patient plasma. Thus, there is now evidence for dysregulation of four neuromodulators (GABA, GHB, glu, gly) in SSADHD biofluids, although we have not documented an age-dependent correlation for glu.

We recently characterized archival newborn screening DBS from patients with SSADHD [4]. Since GHB and GABA are not quantified on any US state newborn screening panel, we sought to identify a metabolic "footprint" suggestive of SSADHD. Using an algorithm measuring the same metabolites as in the current study, informative biomarkers were identified when contrasted with the median and 10th, 25th, 50th, 75th and 90th-centiles of parallel control metabolites. Informative biomarkers (at or below the 1st-centile) included C2-, C3-, C4-, C4OH-carnitines, orn and cre. For post newborn SSADHD DBS, informative biomarkers included $\mathrm{C} 2$ - and $\mathrm{C} 4 \mathrm{OH}$ carnitines, orn, his, and cre. Overall, results for amino acids in the current study (orn, pro, gln (Fig. 3), short-chain acylcarnitines (Fig. 

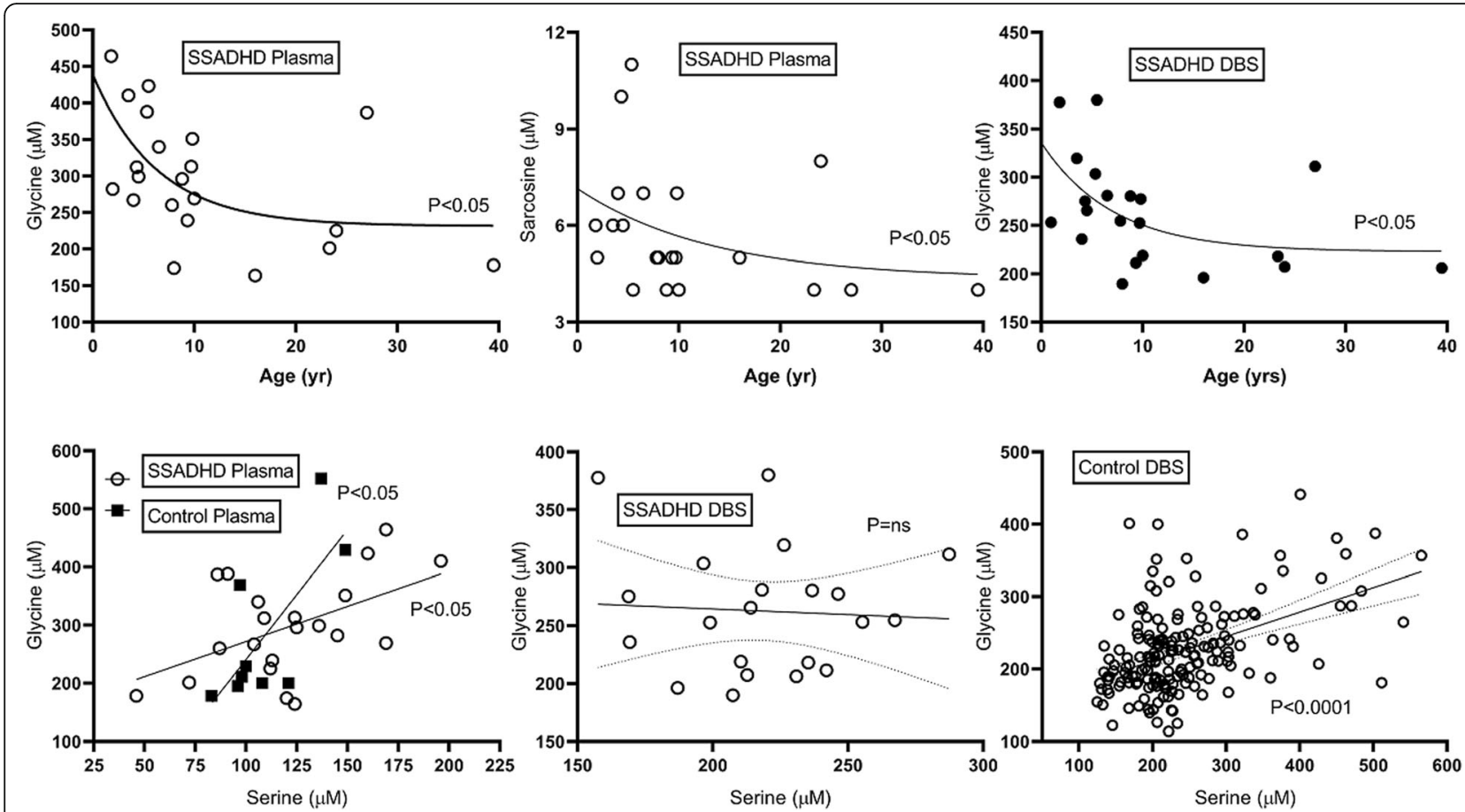

Fig. 2 Amino acid correlations with age for patients and controls in plasma and DBS. Statistical analyses employed either the Pearson correlation or the Spearman ranked correlation

4), and medium-chain acylcarntines (Suppl. Fig. 1)) agreed well with these earlier studies [4]. The finding of significantly decreased ASA, a urea cycle intermediate, is consistent with low orn. Low glutamine (gln) in DBS (Fig. 3 ) is in-line with multiple studies on SSADHD, including human and animal models, and suggests disruption of the glutamine-glutamate-GABA cycle (GGG [3, 5, 7, 11];). The current study, however, highlighted several novel biomarkers, including pro, suac, and figlu, as well as significant decreases of long-chain acylcarnitines (C14-, C16- and C18- species). Further, when measured in regional extracts of autopsied SSADHD brain ([11]; online supplementary data), long-chain acylcarnitines were consistently below the median and quartile reference ranges, especially in frontal and parietal cortices, pons, and hippocampus.
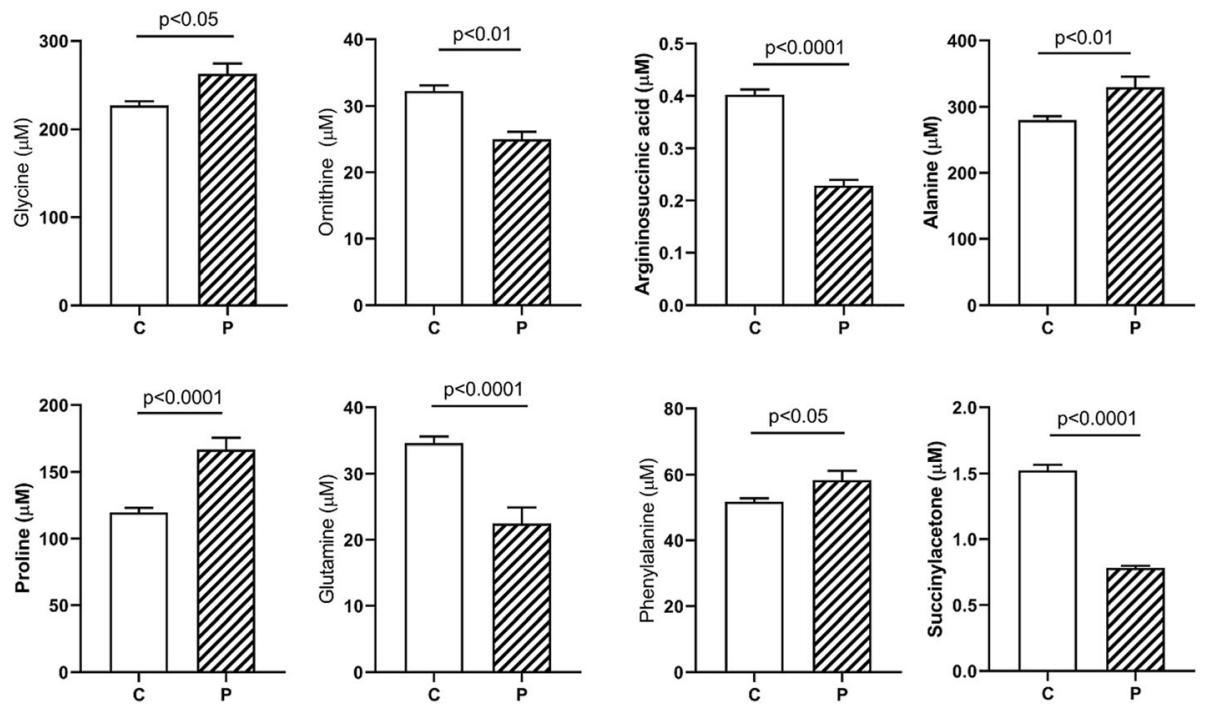

Fig. 3 Abnormal amino acids in DBS for controls (C) and patients (P). Data depicted as mean $\pm \underline{S E M}$. Statistical analysis employed a two-tailed $t$ test 

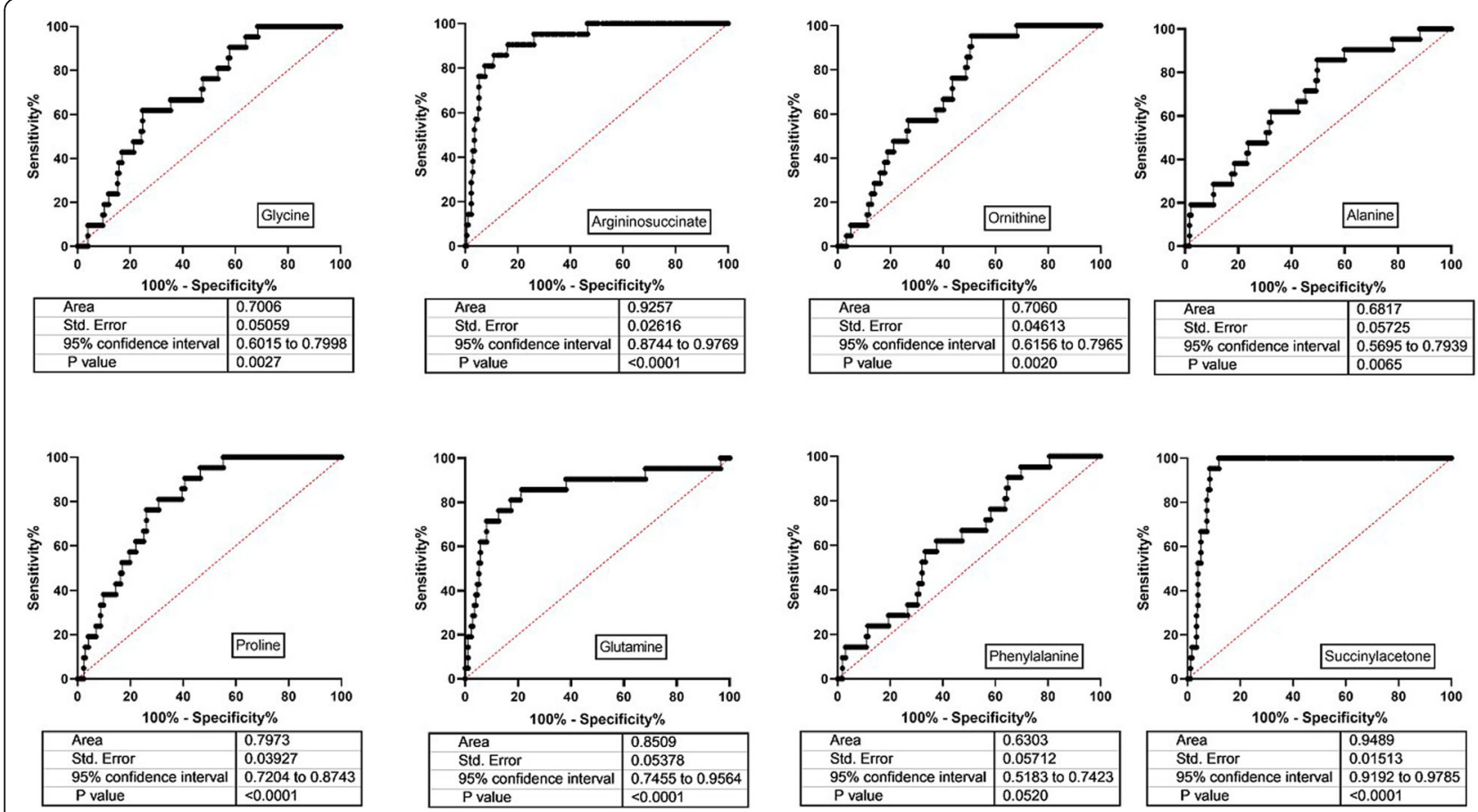

Fig. 4 ROC curves corresponding to the amino acid data shown in Fig. 3

Twenty three acylcarnitines were significantly decreased and only C5:1 carnitine significantly increased. Considering that GABA degradation impacts the Krebs cycle via entry of succinic acid (end-product of GABA catabolism), as well as removal of $\alpha$-ketoglutarate (the nitrogen acceptor for transamination of GABA to succinic semialdehyde), it is reasonable that the $\beta$-oxidation would be enhanced to provide acetyl-CoA to replenish Krebs cycle function. The C5:1 carnitine species arises from the metabolism of leucine, which converts $\beta$ -
methylcrotonyl-CoA to acetoacetate and aceytyl-CoA, and tiglyl-CoA to propionyl-CoA, respectively. GHB itself, or a GHB metabolite (3-oxo-4-hydroxybutyrate, 4hydroxycrotonate) may inhibit both metabolic sequences based upon structural similarities, either as free acid salts or the associated coenzyme A ester [2]. This would explain elevated C5:1 carnitine, supported by the concomitant decrease of C3-carnitine (propionyl-carnitine), the end-product of isoleucine metabolism in which tiglyl$\mathrm{CoA}$ is an intermediate.
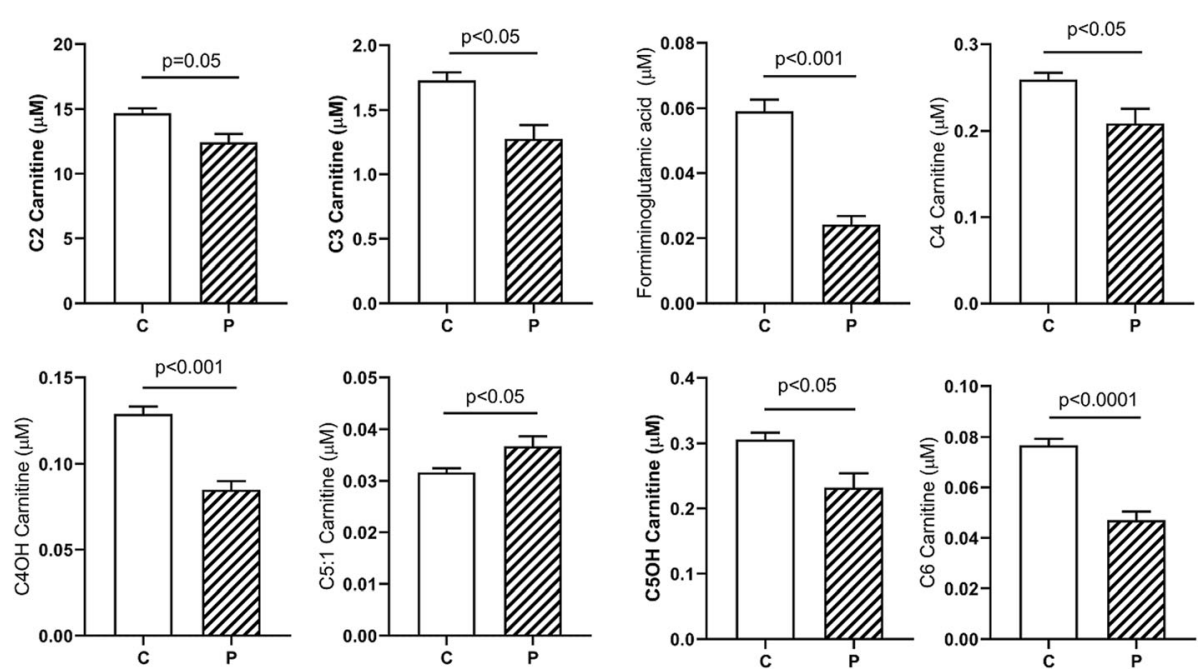

Fig. 5 Abnormal short-chain acylcarnitines in DBS for controls (C) and patients (P). Data depicted as mean + SEM. Statistical analysis employed a two-tailed $t$ test 

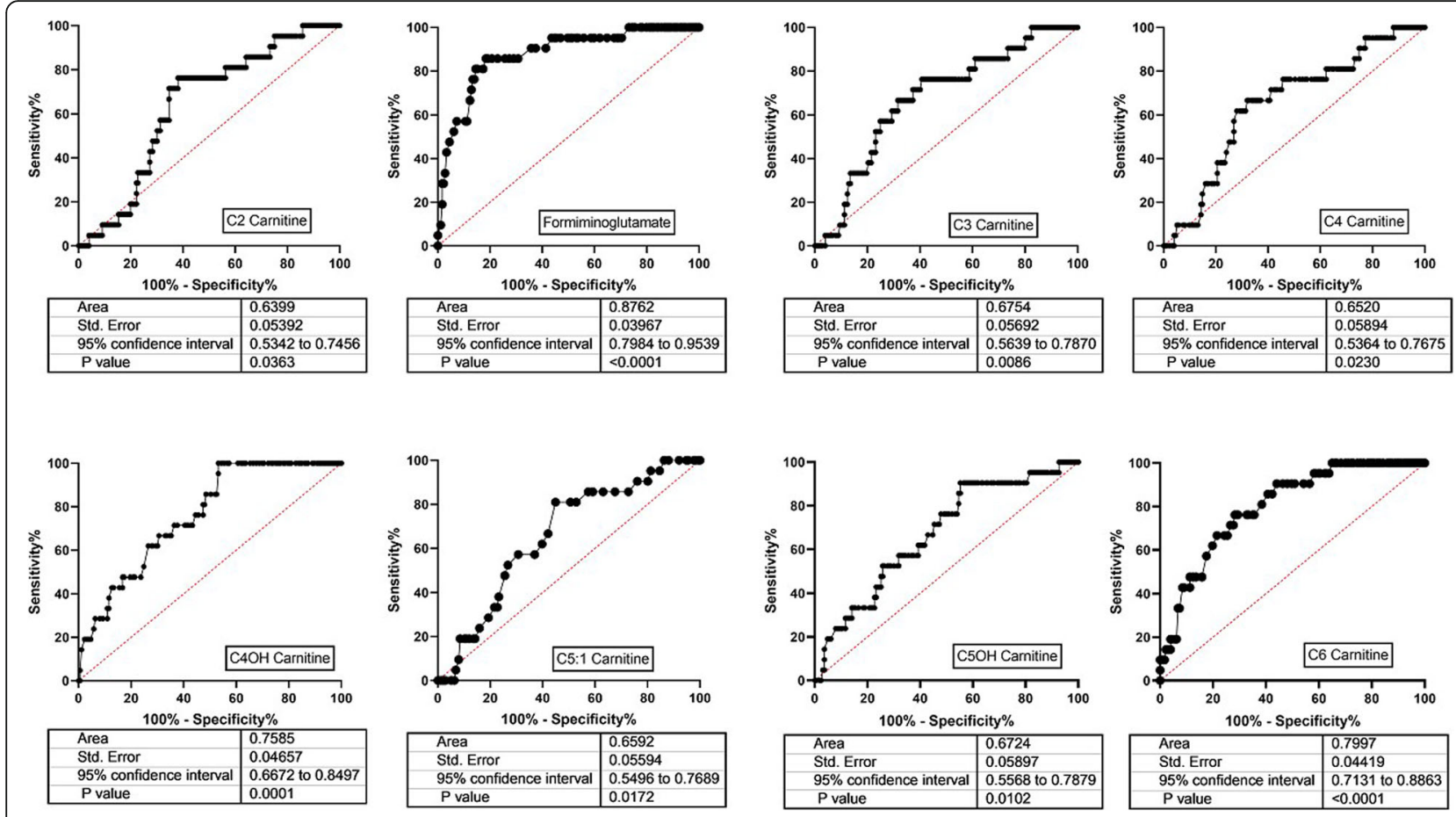

Fig. 6 ROC curves corresponding to the acylcarnitine data shown in Fig. 5

We identified guac and crn as biomarkers of SSADHD in DBS (Suppl. Fig. 7). Low crn is consistent with low crn identified in previous SSADHD post-newborn DBS assessing the potential for newborn screening of SSADHD [4]. Elevation of guac has previously been reported in biofluids of patients and tissue extracts of the murine model [9], and regional brain extracts of autopsied SSADHD brain also revealed elevated guac [11]. The spontaneous non-enzymatic interconversion of cre to crn may offer an explanation of why we did not observe changes in cre levels in the currentstudy.

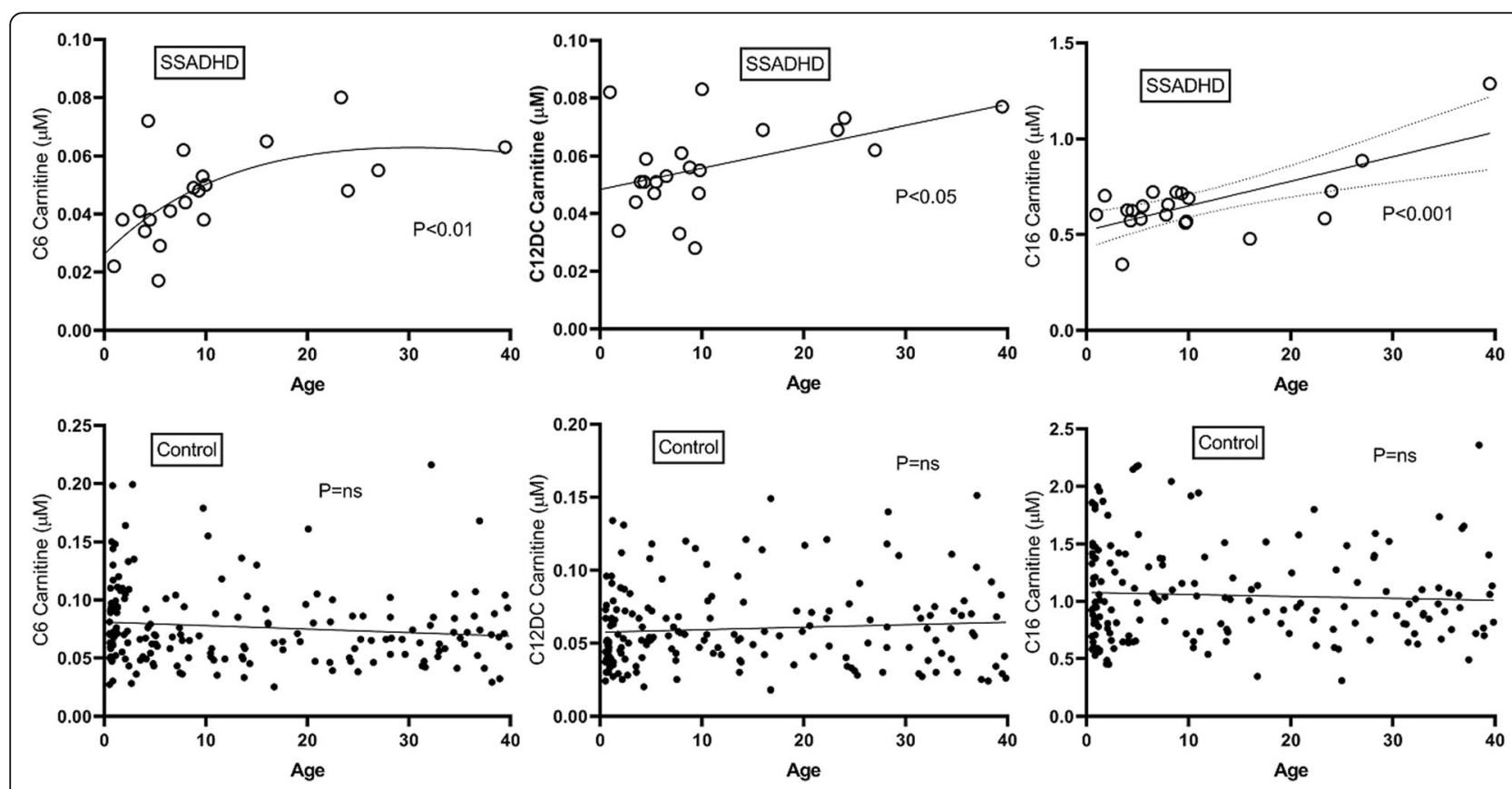

Fig. 7 Correlation of C6-, C12DC (dicarboxylic)-, and C16-carnitines with age in DBS for patients and controls. Statistical analyses employed either the Pearson correlation or the Spearman ranked correlation. Abbreviation: ns, not significant 
Our natural history study of SSADHD, with enrollment of patients of any age, offers the opportunity for multiple age-related metabolic correlations. Along those lines, we evaluated age-dependent correlations for all metabolites characterized. In addition to negative correlations for gly and sarc, we found age-dependent positive correlations ((C6-, C12DC-, C16-, C16:1-, C18:1-, C18:2OH-carnitines) in the SSADHD cohort that were absent in controls. Whether these correlations represent compensatory changes responsive to those for gly, sarc, GHB, and GABA remains to be evaluated. On the other hand, one metabolite seemingly pathognomonic for SSADHD is the sixcarbon 4,5-dihydroxyhexanoate (DHHA, or solerol (sol) when lactonized; Fig. $8[1,11]$;). Metabolic sequences involved in the metabolism/accretion of those acylcarnitines for which age-related positive correlations in patients were identified may be susceptible to inhibition by 4,5-DHHA (Fig. 8) but this too requires further study.

One limitation of the current study is the inability to control for medications in our patient population. Symptomatic interventions in this population usually address neuropsychiatric morbidity (lamotrigine, zonisamide, topiramate, benzodiazepines as needed) but the use of valproate is generally contraindicated. The impact of these medications on the biochemical disturbances observed is not fully known.
Our studies confirm that, in addition to disruptions of the GGG cycle, the GABA shunt, and oxidative phosphorylation, SSADHD appears to significantly alter $\beta$-oxidation, urea and creatine cycles, the sarcosine pathway, as well as multiple amino acid pathways [2, 5, 14]. GABA can cyclize to 2-pyrrolidone, its internal $\gamma$-lactam, a recently described biomarker for GABA-transaminase deficiency [12]. We postulate that 2-pyrrolidone (2-pyr) may provide the link between some of the novel biomarkers identified in the current study, including crn, pro, and figlu (Fig. 8). The structural similarity of 2-pyr to the imidazole/pyrrolidine rings of his, crn and pro, may lead to interference with those metabolic sequences. Similarly, we speculate that interference of tyrosine metabolism at the level of 4maleylacetoacetate /fumarylacetoacetate leads to decreased levels of suac in DBS (Fig. 8), perhaps by accumulated 4,5DHHA, SSA, or GHB itself.

\section{Conclusions}

We have highlighted a number of novel biomarkers in SSADHD, including crn, pro, figlu, sarc, suac, ASA, and EA, as well as age-dependent correlations for sarc and gly and several acylcarnitine species that appear unique for SSADHD. Of those, we found the most discriminating biomarkers to be figlu (area $>0.87$ ) and suac/ASA,

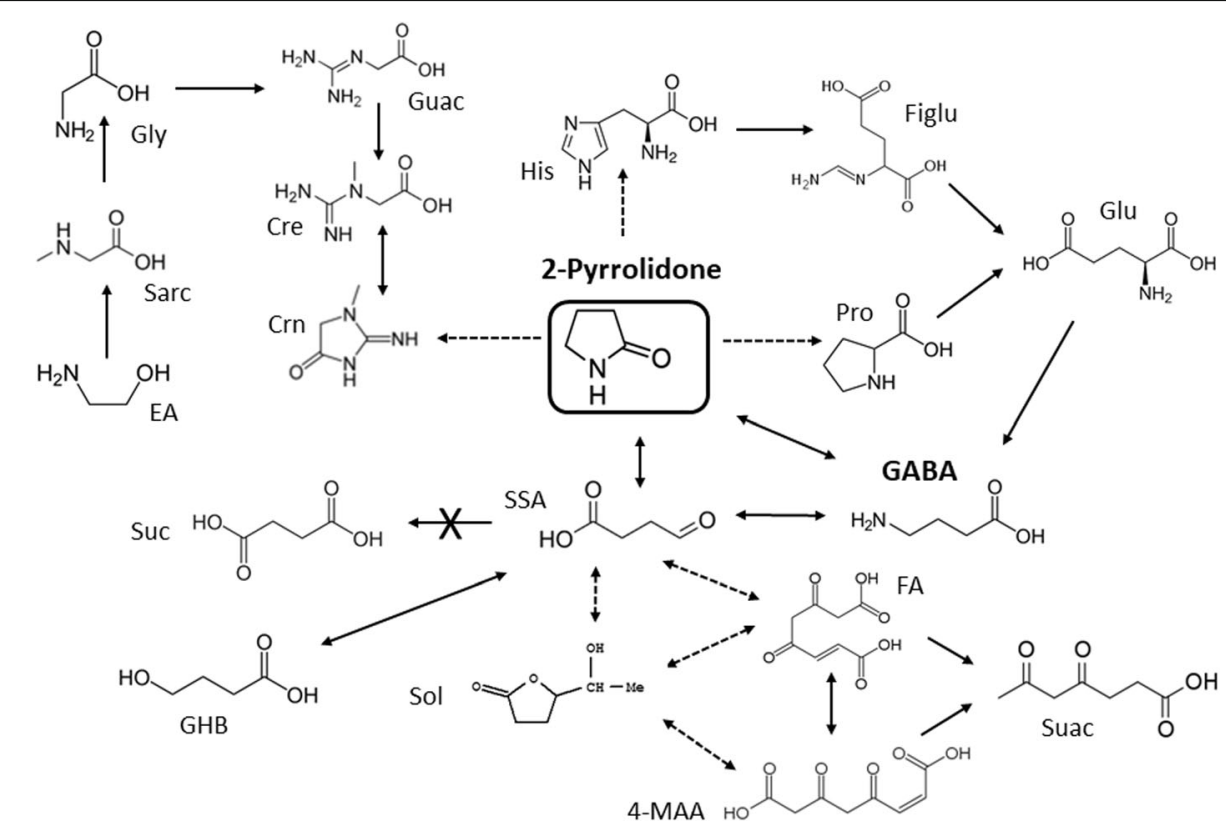

Fig. 8 Potential mechanisms leading to biomarker abnormalities in SSADHD patients. The pathway of GABA metabolism is shown, with X indicating the site of the block in patients with SSADHD. This results in accumulation of GHB, in addition to GABA and succinic semialdehyde (SSA). Under physiological conditions, GABA can undergo lactonization to form the internal $\gamma$-lactam, 2-pyrrolidone (2-pyr [12];). Structural similarities of 2-pyr with the imidazole/pyrrolidine structures of crn, pro and his may associate with alterations in EA, sarc, gly, guac, crn, figlu and pro. Brown et al. [1] first proposed that 4,5-dihydroxyhexanoate (4,5-DHHA; also solerole (sol)) derived from accumulated SSA and an "active" 2carbon fragment (acetyl-CoA, or the acyl- moiety of pyruvic acid). 4,5-DHHA can undergo internal lactonization to at least two species in relation to its vicinal hydroxyl- groups, one of which is solerole. A potential combination of accumulated 4,5-DHHA, sol, SSA or GHB may result in interference with the distal portion of tyrosine metabolism at the level of 4-maleylacetoacetate (4-MAA) and/or fumarylacetoacetate (FA), resulting in decreased Suac. Dashed lines indicate proposed pathways of interference/inhibition 
with areas $>0.92$. We will correlate the age-dependent fluctuation of these biomarkers, along with GABA and GHB, to clinical outcomes (neuroimaging, electrophysiology) in our ongoing natural history study of SSADHD. The objective is to expand insight into the evolving metabolic and clinical pathophysiology of SSADHD, and potentially identify selective metabolic markers that provide predictive insight into the non-specific course of the disease.

\section{Methods}

DBS cards were obtained from twenty-one unique patients with SSADHD (age range 0.95-39.5 years (mean, 11 years; median 8 years); $12 \mathrm{~F}, 9 \mathrm{M}$ ). The samples were obtained at intake in year 01 of our natural history study. SSADHD was previously confirmed through a combination of GHB measurement (urine, blood, DBS), molecular genetic analyses, or assay of SSADH activity in white cells [4]. Parallel control DBS cards were derived from 9 unique individuals (age range 8.4-34.8 years (mean, 18.1 year; median, 16.8 years); $5 \mathrm{~F}, 4 \mathrm{M}$. DBS were obtained using standard finger lance and blood collected onto 903 five spot blood cards (Eastern Business Cards, Greenville, South Carolina). EDTA plasma was obtained from the same patient/control cohorts. Archival reference control data was available $(n=$ 171; age range $0.5-39.9$ years (median, 7.7 years); $80 \mathrm{~F}$, $91 \mathrm{M}$ ) for comparison purposes (https://clir.mayo.edu/).

Amino acids, acylcarnitines, creatine, creatinine, guanidinoacetate, and succinylacetone were quantified in DBS using tandem mass spectrometry as previously described [16]. Amino acids in plasma were quantified by MassTrak analysis as described [17]. Grouped cohorts (patient, control; mean $+/$ - SEM) were evaluated using a two-tailed Student's $t$ test. As this study was exploratory with regard to age, we chose to use univariate statistical analyses without correction for the $p$ value. ROC curves were constructed for all binary comparisons (patient vs. control cohorts). Correlation with age was assessed using the Pearson coefficient or Spearman's ranked test. Significance was set at $p<0.05$. Data was evaluated using GraphPad Prism version 8.0.

\section{Supplementary information}

Supplementary information accompanies this paper at https://doi.org/10. 1186/s13023-020-01522-5.

Additional file 1 Figure S1. Abnormal medium-chain acylcarnitines and ROC curves in DBS of controls (C) and patients (P). Data depicted as mean + SEM. Statistical analysis employed a two-tailed $t$ test.

Additional file 2 Figure S2. Abnormal C14 acylcarnitines and ROC curves in DBS of controls (C) and patients (P). Data depicted as mean \pm SEM. Statistical analysis employed a two-tailed $t$ test.
Additional file 3 Figure $\mathbf{S 3}$. Abnormal $\mathrm{C} 16$ acylcarnitines and ROC curves in DBS of controls (C) and patients (P). Data depicted as mean + SEM. Statistical analysis employed a two-tailed $t$ test.

Additional file 4 Figure S4. Abnormal C18 acylcarnitines in DBS of controls (C) and patients (P). Data depicted as mean \pm SEM. Statistical analysis employed a two-tailed $t$ test.

Additional file $\mathbf{5}$ Figure S5. ROC curves corresponding to the longchain acylcarnitine data shown in Suppl. Fig. 4.

Additional file 6 Figure S6. Correlation of C16:1-, C18:1, and C18:2OHcarnitines with age in DBS for patients and controls. Statistical analyses employed either the Pearson correlation coefficient or the Spearman ranked test. Abbreviation: ns, not significant.

Additional file $\mathbf{7}$ Figure S7. Concentration of $\mathrm{crn}$ and guac in DBS of controls (C) and patients (P). Data are presented as mean + SEM. Data analysis performed using a two-tailed $t$ test.

\section{Abbreviations}

GABA: $\gamma$-aminobutyrate; GHB: $\gamma$-hydroxybutyrate; SSA: Succinic semialdehyde; 4,5-DHHA: 4,5-dihydroxyhexanoate (when lactonized, 4,5-dihydroxyhexanoate Y-lactone; solerole (Sol)); Ala: Alanine; Glu: Glutamate; Gln: Glutamine; Pro: Proline; His: Histidine; Orn: Ornithine; Gly: Glycine; Ser: Serine; Sarc: Sarcosine; Suc: Succinate; EA: Ethanolamine; Guac: Guanidinoacetate; Cre: Creatine; Crn: Creatinine; Suac: Succinylacetone;

Figlu: Formaminoglutamate; GGG: Glutamine-glutamate-GABA cycle; 2-pyr: 2pyrrolidone ( $\gamma$-lactam of GABA); CoA: Coenzyme A; FA: Fumarylacetoacetate; 4-MAA: 4-maleylacetoacetate; ROC curve: Receiver operating characteristic curve

\section{Acknowledgements}

We gratefully acknowledge the participation of patients and family members in the ongoing natural history study of SSADHD.

\section{Authors' contributions}

TK and DCW developed the initial draft of the manuscript and provided data and statistical analysis. XS, MD and PLP obtained specimens and coordinated data analysis. CT and PR were responsible for data development in DBS. EA PA, and TB provided plasma amino acid data. J-BR, MD, PLP, and KMG designed the study and revised secondary and final drafts of the manuscript. All authors read and approved the final manuscript.

\section{Funding}

Supported in part by NIH R01 HD091142 and the support of the SSADH association (www.ssadh.net).

\section{Availability of data and materials}

The datasets used and/or analyzed during the current study are available from the corresponding author upon reasonable request.

\section{Ethics approval and consent to participate}

Collection of biospecimens from SSADHD patients was obtained with informed consent and IRB approval (P00029917) of the Boston Children's Hospital.

\section{Consent for publication}

Not applicable.

\section{Competing interests}

The authors declare that they have no competing interests.

\section{Author details}

${ }^{1}$ Department of Pharmacotherapy, College of Pharmacy and Pharmaceutical Sciences Building Room 210C, Washington State University, 412 E. Spokane Falls Boulevard, Spokane, WA 99202-2131, USA. Mayo Clinic, Department of Laboratory Medicine and Pathology, Rochester, MN, USA. ${ }^{3}$ Baylor Scott \& White Research Institute, Institute of Metabolic Disease, Dallas, TX, USA. ${ }^{4}$ Department of Neurology, Pediatric Neurology, Harvard Medical School and Boston Children's Hospital, Boston, USA. 
Received: 11 May 2020 Accepted: 24 August 2020

Published online: 23 September 2020

\section{References}

1. Brown GK, Cromby CH, Manning NJ, Pollitt RJ. Urinary organic acids in succinic semialdehyde dehydrogenase deficiency: evidence of alphaoxidation of 4-hydroxybutyric acid, interaction of succinic semialdehyde with pyruvate dehydrogenase and possible secondary inhibition of mitochondrial beta-oxidation. J Inherit Metab Dis. 1987;10:367-75.

2. Brown M, Turgeon C, Rinaldo P, Roullet JB, Gibson KM. Temporal metabolomics in dried bloodspots suggests multipathway disruptions in aldh $5 \mathrm{al}^{-/-}$mice, a model of succinic semialdehyde dehydrogenase deficiency. Mol Genet Metab. 2019a;128:397-408.

3. Brown MN, Walters DC, Schmidt MA, Hill J, McConnell A, Jansen EEW, et al. Maternal glutamine supplementation in murine succinic semialdehyde dehydrogenase deficiency, a disorder of $\gamma$-aminobutyric acid metabolism. J Inherit Metab Dis. 2019b;42:1030-9.

4. Brown MN, Turgeon C, Rinaldo P, Pop A, Salomons GS, Roullet J-B, et al. Longitudinal metabolomics in dried bloodspots yields profiles informing newborn screening for succinic semialdehyde dehydrogenase deficiency. JIMD Rep. 2020;53:29-38.

5. Chowdhury GM, Gupta M, Gibson KM, Patel AB, Behar KL. Altered cerebral glucose and acetate metabolism in succinic semialdehyde dehydrogenasedeficient mice: evidence for glial dysfunction and reduced glutamate/ glutamine cycling. J Neurochem. 2007;103:2077-91.

6. DiBacco ML, Roullet JB, Kapur K, Brown MN, Walters DC, Gibson KM, et al. Age-related phenotype and biomarker changes in SSADH deficiency. Ann Clin Transl Neurol. 2018;6:114-20.

7. Gupta M, Polinsky M, Senephansiri H, Snead OC, Jansen EE, Jakobs C, et al. Seizure evolution and amino acid imbalances in murine succinate semialdehyde dehydrogenase (SSADH) deficiency. Neurobiol Dis. 2004;16:556-62.

8. Hirrlinger J, Marx G, Besser S, Sicker M, Köhler S, Hirrlinger PG, et al. GABAglycine cotransmitting neurons in the ventrolateral medulla: development and functional relevance for breathing. Front Cell Neurosci. 2019;13:517.

9. Jansen EE, Verhoeven NM, Jakobs C, Schulze A, Senephansiri H, Gupta M, et al. Increased guanidino species in murine and human succinate semialdehyde dehydrogenase (SSADH) deficiency. Biochim Biophys Acta. 1762;2006:494-8.

10. Jansen EE, Vogel KR, Salomons GS, Pearl PL, Roullet JB, Gibson KM. Correlation of blood biomarkers with age informs pathomechanisms in succinic semialdehyde dehydrogenase deficiency (SSADHD), a disorder of GABA metabolism. J Inherit Metab Dis. 2016;39:795-800.

11. Kirby T, Walters DC, Brown M, Jansen E, Salomons GS, Turgeon C, et al. Postmortem tissue analyses in a patient with succinic semialdehyde dehydrogenase deficiency (SSADHD). I Metabolomic outcomes Metab Brain Dis. 2020;35:601-14.

12. Koenig MK, Bonnen PE. Metabolomics profile in ABAT deficiency pre- and post-treatment. JIMD Rep. 2019;43:13-7.

13. Raposo Pereira F, McMaster MTB, Schellekens A, Polderman N, de Vries YDAT, van den Brink W, et al. Effects of recreational GHB use and multiple GHB-induced comas on brain structure and impulsivity. Front Psychiatry. 2020;11:166.

14. Sauer SW, Kölker S, Hoffmann GF, Ten Brink HJ, Jakobs C, Gibson KM, et al. Enzymatic and metabolic evidence for a region specific mitochondrial dysfunction in brains of murine succinic semialdehyde dehydrogenase deficiency (Aldh5a1-/- mice). Neurochem Int. 2007;50:653-9.

15. Tsai G, Lane HY, Yang P, Chong MY, Lange N. Glycine transporter I inhibitor, $\mathrm{N}$-methylglycine (sarcosine), added to antipsychotics for the treatment of schizophrenia. Biol Psychiatry. 2004;55:452-6.

16. Turgeon C, Magera MJ, Allard P, Tortorelli S, Gavrilov D, Oglesbee D, et al. Combined newborn screening for succinylacetone, amino acids, and acylcarnitines in dried blood spots. Clin Chem. 2008;54:657-64.

17. Walters DC, Arning E, Bottiglieri T, Jansen EEW, Salomons GS, Brown MN, et al. Metabolomic analyses of vigabatrin (VGB)-treated mice: GABAtransaminase inhibition significantly alters amino acid profiles in murine neural and non-neural tissues. Neurochem Int. 2019;125:151-62.

\section{Publisher's Note}

Springer Nature remains neutral with regard to jurisdictional claims in published maps and institutional affiliations. 